\title{
THE HERMITIAN LEVEL OF COMPOSITION ALGEBRAS
}

\author{
S. PUMPLÜN AND T. UNGER
}

\begin{abstract}
The hermitian level of composition algebras with involution over a ring is studied. In particular, it is shown that the hermitian level of a composition algebra with standard involution over a semilocal ring, where two is invertible, is always a power of two when finite. Furthermore, any power of two can occur as the hermitian level of a composition algebra with nonstandard involution. Some bounds are obtained for the hermitian level of a composition algebra with involution of the second kind.
\end{abstract}

\section{INTRODUCTION}

Let $R$ be a unital, commutative, associative ring, equipped with a non-trivial involution ${ }^{-}$(i.e. an anti-automorphism of period 2). For $x \in R$, we call $\bar{x} x$ a hermitian square. The smallest positive integer $m$ such that -1 is a sum of $m$ hermitian squares in $R$ is called the hermitian level of $(R,-)$, denoted $S_{h}(R,-)$. If no such $m$ exists, we set $S_{h}(R,-)=\infty$. Note that $S_{h}(R,-)$ depends on the involution ${ }^{-}$and not just on the $\operatorname{ring} R$ and also that if ${ }^{-}=\mathrm{id}_{R}$, then $S_{h}\left(R,{ }^{-}\right)=s(R)$ is the usual level.

In [7], Lewis showed that there exist commutative rings with non-trivial involution having any positive integer as hermitian level. He also showed that the hermitian level, if finite, is a power of two for fields $K$ of characteristic not two with non-trivial involution and for quaternion division algebras $D$ over fields of characteristic not two with the standard involution.

$K$ and $D$ are exactly the non-split composition algebras of dimension 2 and 4 over a suitable field $k$. It is well-known that composition algebras only exist in dimension 1, 2, 4 and 8. An 8-dimensional composition algebra $C$ is called an octonion (or Cayley) algebra and, although it is not associative, it retains the most pleasing property of $K$ and $D$ : it comes equipped with a non-degenerate norm form with values in $k$, which is multiplicative.

We investigate the hermitian level of composition algebras $C$ with involution over a ring $R$, extending the results of Lewis [7] for the standard involution ${ }^{-}$and some of the results of Serhir [14] for the non-standard involution ${ }^{\wedge}$ on $C$ (which we introduce here in a more general context). In particular, we show that over semilocal rings where 2 is invertible, the hermitian level of $(C,-)$ is a power of two if it is finite and that all powers of two can occur as the hermitian level of

Date: 21 March 2002.

2000 Mathematics Subject Classification. 17A75, 16W10, 11E25. 
$(C, \widehat{ })$. Finally, we obtain some bounds on the hermitian level of $C$, equipped with an involution of the second kind.

Our methods are mostly quadratic form theoretic in nature and usually adaptations of Lewis's arguments to the more general setting of rings. We refer to the standard works [1], [6] and [12] for background reading on quadratic forms over rings, algebras with involution and nonassociative algebras respectively.

\section{Preliminaries}

2.1. Composition Algebras with Involution. Let $R$ be a unital commutative associative ring, and $A$ a unital nonassociative $R$-algebra which is finitely generated projective of (constant) rank $>0$ as an $R$-module. We will simply call such an algebra $A$ an " $R$-algebra" in the remainder of this paper.

Associativity in $A$ is measured by the associator $[x, y, z]=(x y) z-x(y z)$, commutativity by the commutator $[x, y]=x y-y x$. Define the nucleus of $A$ by

$$
\operatorname{Nuc}(A)=\{x \in A \mid[x, A, A]=[A, x, A]=[A, A, x]\}
$$

and the commuter of $A$ by

$$
\operatorname{Comm}(A)=\{x \in A \mid[x, A]=0\} .
$$

An $R$-algebra $C$ is called a composition algebra, if it carries a quadratic form $n: C \rightarrow R$ satisfying the following two conditions:

(i) Its induced symmetric bilinear form $n(x, y):=n(x+y)-n(x)-n(y)$ is nondegenerate, i.e. determines an $R$-module isomorphism $C \stackrel{\sim}{\longrightarrow} C^{\vee}=$ $\operatorname{Hom}_{R}(C, R)$.

(ii) $n$ permits composition, that is $n(x y)=n(x) n(y)$ for all $x, y \in C$.

A composition algebra over $R$ is split if it contains a composition subalgebra, isomorphic to $R \oplus R$. (See [9] for an explicit description of split composition algebras.)

An $R$-algebra $A$ is called quadratic in case there exists a quadratic form $n: A \rightarrow$ $R$ such that

$$
n\left(1_{A}\right)=1 \quad \text { and } \quad x^{2}-n\left(1_{A}, x\right) x+n(x) 1_{A}=0
$$

for all $x \in A$. The form $n$ is uniquely determined and called the norm of the quadratic algebra $A$.

A nonassociative algebra is called alternative if its associator $[x, y, z]$ is alternating. Composition algebras are quadratic alternative algebras. More precisely, a quadratic form $n$ of the composition algebra satisfying (i) and (ii) above agrees with its norm as a quadratic algebra and thus is unique. It is called the norm of the composition algebra $C$ and is sometimes also denoted by $n_{C}$.

A quadratic alternative algebra is a composition algebra if and only if its norm is nondegenerate $[8,4.6]$. Composition algebras only exist in ranks 1, 2, 4 or 8. Those of rank 2 are exactly the quadratic étale $R$-algebras, those of rank 4 
exactly the well-known quaternion algebras. The ones of rank 8 are also called octonion algebras.

Let $\tau$ be an involution on $A$, i.e. a map $\tau: A \rightarrow A$ satisfying

$$
\tau(x+y)=\tau(x)+\tau(y), \tau(x y)=\tau(y) \tau(x), \text { and } \tau^{2}(x)=x
$$

for all $x, y \in A$.

If 2 is an invertible element in $R$, we have $A=\operatorname{Skew}(A, \tau) \oplus \operatorname{Sym}(A, \tau)$ with

$$
\operatorname{Skew}(A, \tau):=\{x \in A \mid \tau(x)=-x\}
$$

the set of skew-symmetric elements and

$$
\operatorname{Sym}(A, \tau):=\{x \in A \mid \tau(x)=x\}
$$

the set of symmetric elements in $A$ with respect to $\tau$. An involution is called scalar if all the norms $\tau(x) x$ are scalars in $R$, and hence by linearization all traces $\tau(x)+x$ are scalars in $R$, too. Note that for a scalar involution, $n_{A}(x)=\tau(x) x$ resp. $t_{A}(x)=\tau(x)+x$ is a quadratic resp. a linear form on $A$, whenever $a 1_{A}=0$ implies $a=0$, for every $a \in R[8$, p. 86].

An involution $\tau$ on $A$ is called of the first kind if $\left.\tau\right|_{R}=$ id. Let $S$ be a quadratic étale $R$-algebra with standard involution $s_{0}$, and let $A$ be an algebra over $S$. An involution on $A$ whose restriction to $S$ is the standard involution is called of the second kind.

Given an $R$-algebra $A$ together with an involution $\tau$, an element in $A$ of the form $\tau(x) x$ with $x \in A$ is called a hermitian square in $A$. Following [7] the hermitian level of $A$ is the least integer $m$ such that

$$
-1=\tau\left(x_{1}\right) x_{1}+\cdots+\tau\left(x_{m}\right) x_{m} .
$$

We write $S_{h}(A, \tau)$ for the hermitian level and define $S_{h}(A, \tau)=\infty$ if -1 cannot be written as a sum of hermitian squares in $(A, \tau)$. Obviously, the hermitian level of an algebra depends on the particular involution and not just on the algebra.

Let $a_{i} \in R^{\times}(1 \leq i \leq m)$ be invertible. We define a map $h: A^{m} \times A^{m} \rightarrow A$ by

$$
h(x, y):=a_{1} \tau\left(x_{1}\right) y_{1}+\cdots+a_{m} \tau\left(x_{m}\right) y_{m}
$$

for $x=\left(x_{1}, \ldots, x_{m}\right), y=\left(y_{1}, \cdots, y_{m}\right) \in A^{m}$. This map is biadditive and satisfies

$$
h(x \alpha, y \beta)=a_{1} \tau\left(x_{1} \alpha\right)\left(y_{1} \beta\right)+\cdots+a_{m} \tau\left(x_{m} \alpha\right)\left(y_{m} \beta\right)=\tau(\alpha) h(x, y) \beta
$$

provided that $R 1_{A} \subset \operatorname{Comm}(A) \cap \operatorname{Nuc}(A)$. The map $h$ is called isotropic if there are $x_{1}, \ldots, x_{m} \in A$ with at least one invertible $x_{i} \in A^{\times}$, such that

$$
\sum_{i=1}^{m} a_{i} \tau\left(x_{i}\right) x_{i}=0,
$$

otherwise it is called anisotropic. Write $m \times\langle 1\rangle$ for the map $h: A^{m} \times A^{m} \rightarrow A$, represented by the quadratic form $\langle 1, \ldots, 1\rangle=m \times\langle 1\rangle$, i.e. for $h(x, y)=\tau\left(x_{1}\right) y_{1}+$ $\cdots+\tau\left(x_{m}\right) y_{m}$. 
A composition algebra $C$ comes endowed with a standard involution ${ }^{-}$, given by $\bar{x}=t(x) 1_{C}-x$, where $t: C \rightarrow R$ is the trace map given by $t(x):=n\left(1_{C}, x\right)$. This involution is scalar.

2.2. The Cayley-Dickson Doubling Process. The Cayley-Dickson doubling process is a well-known way to construct new algebras with scalar involution out of a given one with such an involution. Let $A$ be an $R$-algebra with involution $*$ and let $\mu \in R$ be such that $\mu x=0$ implies $x=0$ in $A$. Then the $R$-module $A \oplus A$ becomes a unital algebra via the multiplication

$$
(u, v)\left(u^{\prime}, v^{\prime}\right)=\left(u u^{\prime}+\mu v^{\prime *} v, v^{\prime} u+v u^{\prime *}\right)
$$

for $u, u^{\prime}, v, v^{\prime} \in A$, with involution

$$
(u, v)^{*}=\left(u^{*},-v\right) .
$$

It is called the (classical) Cayley-Dickson doubling of $A$, and denoted by Cay $(A, \mu)$. The involution $*$ is a scalar involution on $\operatorname{Cay}(A, \mu)$ if and only if $*$ is a scalar involution on $A$, with norm

$$
n_{\mathrm{Cay}(A, \mu)}((u, v))=n_{A}(u)-\mu n_{A}(v) .
$$

The Cayley-Dickson doubling process depends on the scalar $\mu$ only up to an invertible square. By repeated application of the Cayley-Dickson doubling process, starting from a composition algebra $C$ over $R$, we obtain either again a composition algebra (if the rank of the new algebra is less than or equal to 8), or a generalized Cayley-Dickson algebra of rank $2^{m} \operatorname{rank}(C) \geq 16$. The latter are no longer alternative, but still flexible (i.e. $(x y) x=x(y x)$ holds for all $x, y$ in the algebra) with a scalar involution (cf. [8]).

When iterating the Cayley-Dickson doubling process, we use the shorthand notation

$$
\operatorname{Cay}(A, \mu, \nu):=\operatorname{Cay}(\operatorname{Cay}(A, \mu), \nu) \text {. }
$$

Over fields, the classical Cayley-Dickson process indeed generates all possible composition algebras. Over rings, a more general version is required, which still does not always yield all possible composition algebras, only those containing a composition subalgebra of half its rank. This "generalized" Cayley-Dickson doubling process is due to Petersson [9]. Its description is quite technical. Here is the main result:

Let $D$ be a composition algebra over $R$ of rank $\leq 4$ with standard involution - . Let $P$ be a finitely generated projective right $D$-module of rank one, carrying a nondegenerate hermitian form $h: P \times P \rightarrow D$. Define $N: P \rightarrow D$ by $N(w):=$ $h(w, w)$ for $w \in P$. For each $\mu \in R$, the $R$-module

$$
A=D \oplus P
$$

becomes a new $R$-algebra via the multiplication

$$
(u, w)\left(u^{\prime}, w^{\prime}\right)=\left(u u^{\prime}+\mu h\left(w^{\prime}, w\right), w^{\prime} \cdot u+w \cdot \bar{u}^{\prime}\right)
$$


for $u, u^{\prime} \in D, w, w^{\prime} \in P$, and where denotes the right $D$-module structure of $P$. The algebra $A$ constructed above is denoted by $\operatorname{Cay}(D, P, \mu N):=\operatorname{Cay}(D, P, \mu h)$. $D$ itself is canonically a (free) right $D$-module of rank one and "norm one" (cf. [9]). Any norm on $D$ is similar to $n_{D}$ (resp. any nondegenerate hermitian form $h: D \times D \rightarrow D$ is similar to the canonical form given by the involution, i.e., by $\left.\left(w, w^{\prime}\right) \mapsto \bar{w}^{\prime} w\right)$. In this special case the "classical" Cayley-Dickson doubling $\operatorname{process} \operatorname{Cay}(D, \mu):=\operatorname{Cay}\left(D, D, \mu n_{D}\right)$ with $\mu \in D$ is obtained.

\section{The Standard Involution}

When investigating the hermitian level, one of the easiest classes of nonassociative algebras to look at are algebras $A$ with a scalar involution, since for these the hermitian square $\tau(x) x$ is an element in $R$, and the map $x \rightarrow \tau(x) x$ a quadratic form, provided that the ring $R$ is isomorphic to a subring of $A$ (cf. [8]). We start our investigation by looking at composition algebras with standard involution -

If $k$ is a field of characteristic not two, and $C$ a split composition algebra over $k$ with standard involution ${ }^{-}$, then it is easy to see that $-1=\bar{x} x$ for an element $x \in\{y \in C \mid t(y)=0\}=C_{0}$, i.e. for a pure quaternion (resp. octonion) in case $C$ is a quaternion (resp. octonion) algebra. More generally, we obtain:

Lemma 3.1. Let $R$ be a ring with $2 \in R^{\times}$, and $C$ a split composition algebra over $R$ with standard involution ${ }^{-}$, then $S_{h}(C,-)=1$.

Proof. $C$ is split if and only if $C$ contains the split quadratic étale algebra $R \oplus R$ with hyperbolic norm $n \simeq\langle 1,-1\rangle$, in particular, $n_{C} \simeq\langle 1,-1\rangle \perp \cdots$ which implies that $n_{C}(x)=\bar{x} x=-1$ for a suitable element $x \in C$.

Note that any quaternion algebra over $R$ with norm $n_{C} \simeq\langle 1,-1\rangle \perp \cdots$ splits, but that this is not necessarily so for an octonion algebra.

Lemma 3.2. Let $R$ be a ring and let $C$ be a composition algebra over $R$ with standard involution - .

(1) If $S_{h}(C,-)=m$, then the form $(m+1) \times n_{C}$ is isotropic. Moreover, if $m \times n_{C}$ already is isotropic, then every isotropic vector $\left(x_{1}, \ldots, x_{m}\right)$ satisfies $n_{C}\left(x_{i}\right)=\bar{x}_{i} x_{i} \notin R^{\times}$(or, equivalently, $\left.x_{i} \notin C^{\times}\right)$, for all $i, 1 \leq$ $i \leq m$.

(2) Let $m \times n_{C}$ be anisotropic and let $\left(x_{1}, \ldots, x_{m+1}\right)$ be an isotropic vector of $(m+1) \times n_{C}$ such that $x_{i} \in C^{\times}$for all $i, 1 \leq i \leq(m+1)$, then $S_{h}\left(C,{ }^{-}\right)=$ $m$.

Proof. (1) Let $m$ be the smallest integer such that $-1=\bar{x}_{1} x_{1}+\cdots+\bar{x}_{m} x_{m}$. Then $0=1+\bar{x}_{1} x_{1}+\cdots+\bar{x}_{m} x_{m}$, hence $(m+1) \times n_{C}$ is isotropic. In case $m \times n_{C}$ is isotropic, and there exists an isotropic vector $\left(x_{1}, \ldots, x_{m}\right)$ where $x_{i}$ is invertible in $C$, for some $i, 1 \leq i \leq m$, then $0=\bar{x}_{1} x_{1}+\cdots+\bar{x}_{m} x_{m}$. Without loss of generality assume $x_{1} \in C^{\times}$. Then also $\bar{x}_{1} \in C^{\times}$and $0=1+\left(\overline{x_{1}^{-1} x_{2}}\right)\left(x_{1}^{-1} x_{2}\right)+$ $\left.\cdots+\overline{\left(x_{1}^{-1} x_{m}\right.}\right)\left(x_{1}^{-1} x_{m}\right)$, contradicting the minimality of $m$. 
(2) Let $(m+1) \times n_{C}$ be isotropic. Then as above this implies $S_{h}\left(C,{ }^{-}\right) \leq$ $m$, provided that there exists an isotropic vector $\left(x_{1}, \ldots, x_{m+1}\right)$ such that $x_{i}$ is invertible in $C$, for some $i, 1 \leq i \leq m+1$. Let $s=S_{h}\left(C,{ }^{-}\right)$, then $-1=$ $\bar{x}_{1} x_{1}+\cdots+\bar{x}_{s} x_{s}$ and $(s+1) \times n_{C}$ is isotropic. Since $m \times n_{C}$ is anisotropic, we must have $m<s+1$ and conclude that $S_{h}(C,-)=m$.

Note that for any algebra with involution $(A, \tau), S_{h}(A, \tau)=m$ always implies that the "pseudo-hermitian form" $(m+1) \times\langle 1\rangle$, as defined in 2.1, is isotropic. However, the multiplicativity of the norm and the fact that the standard involution of a composition algebra is scalar are crucial in the converse of the above lemma.

In particular, for semilocal rings we obtain a generalization of $[7,1.1]$ (applying $[1$, III.(5.2)(iii)] as for instance in [1, III.(5.3), p. 87]):

\section{Corollary 3.3.}

(1) Let ${ }^{-}$be the standard involution on a composition algebra $C$ of rank greater than 1 over a semilocal ring $R$ with $2 \in R^{\times}$. Let $m \geq 2$ and assume that either $m$ is even, or that $|R / \mathfrak{m}| \geq 3$, for all $\mathfrak{m} \in \max (R)$. Then $S_{h}\left(C,{ }^{-}\right)=m$ if and only if $m \times n_{C}=\langle 1, \ldots, 1\rangle \otimes n_{C}$ (m-times) is anisotropic, but $(m+1) \times n_{C}$ is isotropic.

(2) Let $^{-}$be the standard involution on a composition division algebra $C$ over a field $k$ of $\operatorname{char}(k) \neq 2$. Then $S_{h}\left(C,{ }^{-}\right)=m$ if and only if $m \times n_{C}=$ $\langle 1, \ldots, 1\rangle \otimes n_{C}$ (m-times) is anisotropic, but $(m+1) \times n_{C}$ is isotropic.

Lemma 3.4 (cf. $[7,1.2]$ ). Let $C$ be a composition division algebra over a field $k$ of $\operatorname{char}(k) \neq 2$ with standard involution -. The following are equivalent:

(1) Zero is a nontrivial sum of hermitian squares, i.e. there exists an integer $m$ such that $m \times n_{C}$ is an isotropic quadratic form.

(2) -1 is a sum of hermitian squares.

(3) Each symmetric element of $C$ is a sum of hermitian squares.

Proof. The above corollary implies the equivalence of (1) and (2).

$(3) \Rightarrow(2)$ is obvious, since $-1 \in \operatorname{Sym}\left(C,{ }^{-}\right)$.

$(1) \Rightarrow(3)$ : Let $0=\bar{x}_{1} x_{1}+\cdots+\bar{x}_{m} x_{m}=n_{C}\left(x_{1}\right)+\cdots+n_{C}\left(x_{m}\right)$ with $x_{i} \in C$ not all zero. Then $m \times n_{C}$ is an isotropic $k$-quadratic form, hence universal, and represents all invertible elements $a \in \operatorname{Sym}\left(C,{ }^{-}\right)$. That is, for any $a \in k^{\times}$there are $x_{i} \in C$ such that $a=n_{C}\left(x_{1}\right)+\cdots+n_{C}\left(x_{m}\right)$.

The conclusion that (3) implies (2) implies (1) in the above proof also holds for composition algebras over rings $R$ where 2 is invertible. The proof of (1) implies (3) holds provided that the following is true: If the $R$-quadratic form $\langle 1, \ldots, 1\rangle \otimes n_{C}$ is isotropic it contains a hyperbolic plane.

The following generalizes $[7,1.5]$.

Proposition 3.5. Let $C$ be a composition algebra over a semilocal ring $R$ with $2 \in R^{\times}$, such that $n_{C}=\langle\langle a, b\rangle\rangle$ or $n_{C}=\langle\langle a, b, c\rangle\rangle$ is a Pfister form (this is always 
the case if $C$ has rank $\leq 4)$. If $S_{h}\left(C,{ }^{-}\right)$is finite then $S_{h}\left(C,{ }^{-}\right)$is a power of 2 . More precisely, then $S_{h}(C,-)=2^{-1} d$ with $d$ the order of the norm $n_{C}$ in $W(R)$, the Witt group of non-singular quadratic forms over $R$.

Proof. Assume that $S_{h}(C,-)=m$ is finite. Then $-1=\bar{x}_{1} x_{1}+\cdots+\bar{x}_{m} x_{m}=$ $n_{C}\left(x_{1}\right)+\cdots+n_{C}\left(x_{m}\right)$ and $(m+1) \times n_{C}$ is isotropic. Pick $s$ such that $2^{s}<$ $m+1 \leq 2^{s+1}$. Then $2^{s+1} \times n_{C} \cong\langle 1, \ldots, 1\rangle \otimes n_{C}$ is an isotropic Pfister form, thus hyperbolic [1, IV.(3.2)]. Therefore

$$
2^{s+1} \times n_{C} \cong\langle\langle-1, \ldots,-1\rangle\rangle \cong\langle 1,-1, \ldots, 1,-1\rangle \otimes n_{C}
$$

and by Witt cancellation, $\langle-1, \ldots,-1\rangle \otimes n_{C} \cong\langle 1, \ldots, 1\rangle \otimes n_{C}$. It follows that $-1 \in D\left(2^{s} \times n_{C}\right)$, i.e. -1 is represented by $2^{s} \times n_{C}$, and so $m=2^{s}$.

Moreover, obviously $n_{C}$ is a torsion space whose order $d$ in $W(R)$ is less than or equal to $2^{s+1}$. Now $d$ must be a power of $2[1,(6.1)$, p. 143], hence even $d=2^{s+1}$, since $2^{s} \times n_{C} \sim 0$ implies that -1 is a sum of $2^{s-1}<m$ hermitian squares in $(C,-)$, a contradiction. We conclude that $m=2^{-1} d$.

Corollary 3.6. Let $C$ be a composition (division) algebra over a field $k$ of $\operatorname{char}(k) \neq 2$. If $S_{h}(C,-)$ is finite then $S_{h}(C,-)$ is a power of 2. More precisely, then $S_{h}\left(C,{ }^{-}\right)=2^{-1} d$ with $d$ the order of the norm $n_{C}$ in $W(k)$.

Even more general, let $A$ be a generalized Cayley-Dickson algebra of rank $2^{r}$ over a semilocal ring $R$ with 2 invertible in the sense of $[8,(6.2)]$, obtained from $R 1$ by a repeated application of the (classical) Cayley-Dickson doubling process with scalar involution $*$ as described in $[8,(6.3)]$. Then $S_{h}(A, *)$ also is a power of two if it is finite, by the same argument as in the above proof.

An immediate consequence of the last proposition is the fact that any prescribed power of two occurs as the level of an octonion division algebra with standard involution over a field (cf. [7, Comment 2, p.470]). Let

$$
k=\mathbb{R}\left(x_{1}, \ldots, x_{m}\right)\left(\sqrt{-\sum_{i=1}^{m} x_{i}^{2}}\right)
$$

be a field of level $s(k)=S_{h}(k$, id $)=2^{s}$. If $s(k) \geq 8$, then the octonion algebra Cay $(k,-1,-1,-1)$ with standard involution has hermitian level $2^{s-3}$.

Recall that the level $s(R)$ of a semilocal ring $R$, where 2 is invertible, is either infinite or a power of two $[1,($ A.3), p. 177]. The following analog to $[7,(1.7)]$ holds in the setting of semilocal rings (it even holds for a generalized Cayley-Dickson algebra obtained from $R 1$ with scalar involution $*$ ):

Proposition 3.7 ([4, (1.5)], [1, (2.4), p. 95]). Let $R$ be a semilocal ring where 2 is invertible. Let $C$ be a composition algebra over $R$ with standard involution whose norm $n_{C}$ is a Pfister form. Let $t$ be a positive integer. The set of invertible values represented by the quadratic form $n_{C} \otimes\langle\langle 1, \ldots, 1\rangle\rangle=2^{t} \times n_{C}$ over $R$ is a group. 
However, we can do better than this:

Proposition 3.8 ([13, 5.6]). Let $R$ be a semilocal ring where 2 is invertible. Let $C$ be a composition algebra over $R$ with standard involution ${ }^{-}$whose norm $n_{C}$ is a Pfister form. Let $m=2^{t}$. For every $\sum_{i=1}^{m} \bar{z}_{i} z_{i} \in R^{\times}, \sum_{i=1}^{m} \bar{w}_{i} w_{i} \in R$ there exist $u_{1}, \ldots, u_{m} \in C$ such that

$$
\left(\sum_{i=1}^{m} \bar{z}_{i} z_{i}\right)\left(\sum_{i=1}^{m} \bar{w}_{i} w_{i}\right)=\sum_{i=1}^{m} \bar{u}_{i} u_{i} .
$$

Moreover, there are $v_{1}, \ldots, v_{m} \in C$ such that

$$
\left(\sum_{i=1}^{m} \bar{z}_{i} z_{i}\right)\left(\sum_{i=1}^{m} \bar{v}_{i} v_{i}\right)=\sum_{i=1}^{m} \bar{w}_{i} w_{i} .
$$

Corollary 3.9 (cf. $[7,1.9]$ ). Let $m=2^{s}$ be a power of two. Let $A$ be a generalized Cayley-Dickson algebra over $R$ of rank $2^{s}$ in the sense of $[8,(6.1),(6.2)]$ obtained from $R 1$ by a repeated application of the (classical) Cayley-Dickson doubling process with scalar involution $*$.

(1) Let $R$ be a semilocal ring where 2 is invertible. Then the product of two sums of $m$ hermitian squares in $(A, *)$, where one is an invertible element in $R$, is again a sum of $m$ hermitian squares. In particular: Let $(C,-)$ be a composition algebra over $R$. Then the product of two sums of $m$ hermitian squares in $(C,-)$, where one is an invertible element in $R$, is again a sum of $m$ hermitian squares.

(2) Let $k$ be a field of $\operatorname{char}(k) \neq 2$. Then the product of two sums of $m$ hermitian squares in $(A, *)$ is again a sum of $m$ hermitian squares. In particular: Let $\left(C,{ }^{-}\right)$be a composition algebra over $k$. Then the product of two sums of $m$ hermitian squares in $(C,-)$ is again a sum of $m$ hermitian squares.

Proof. (1) For $m=2^{s}$, the form $m \times n_{A}$ with $n_{A}(x) 1_{A}=x^{*} x$ (and in particular, $\left.m \times n_{C}\right)$ is a Pfister form, and thus round [1, IV.(2.4), p.95], meaning that the set $D\left(m \times n_{A}\right)$ of invertible elements represented by the form is a group under multiplication.

(2) is now obvious.

Note that we can reduce the number of squares needed in the above proposition by one in certain cases (cf. [7, (1.10)]), since the following holds:

Proposition 3.10 ([1, p. 104]). Let $R$ be a semilocal ring where 2 is invertible, and let $C$ be a composition algebra over $R$ with standard involution ${ }^{-}$. Let $z=$ $\left(z_{1}, \ldots, z_{m}\right)$ be such that $\sum_{i=1}^{m} \bar{z}_{i} z_{i} \in R^{\times}$. Then

$$
\left(\sum_{i=1}^{m} \bar{z}_{i} z_{i}\right)\left(\sum_{i=1}^{m} \bar{w}_{i} w_{i}\right)=\sum_{i=1}^{m} \bar{u}_{i} u_{i}
$$


with $u_{1}=\sum_{i=1}^{m} \bar{z}_{i} w_{i}+\sum_{i=1}^{m} \bar{w}_{i} z_{i}=\langle\langle 1, \ldots, 1\rangle\rangle \otimes n_{C}\left(\left(z_{1}, \ldots, z_{m}\right),\left(w_{1}, \ldots, w_{m}\right)\right)$.

Note that a form of type $n_{C} \otimes\langle\langle 1, \ldots, 1\rangle\rangle$ is not round over every ring (cf. [2]), hence the above proofs do not always work. What remains true over an arbitrary ring is the following property (which, dropping the assumption on $\left(z_{1}, \ldots, z_{m}\right)$ is trivially satisfied even for any integer $m$ ):

Let $C$ be a composition algebra with standard involution ${ }^{-}$over a ring $R$. Then the form $n_{C} \otimes\left\langle\left\langle a_{1}, \ldots, a_{m}\right\rangle\right\rangle$ is the norm $n_{A}$ of the algebra

$$
A=\operatorname{Cay}\left(C,-a_{1}, \ldots,-a_{m}\right)
$$

and thus satisfies $n_{A}\left(x^{2}\right)=n_{A}(x)^{2}$ for all $x \in A$.

In particular: The square of a sum of $s=2^{r}$ hermitian squares of a composition algebra $C$ over $R$ with standard involution is again a sum of $s=2^{r}$ hermitian squares, i.e. $\left(\bar{x}_{1} x_{1}+\cdots+\bar{x}_{s} x_{s}\right)^{2}=\bar{z}_{1} z_{1}+\cdots+\bar{z}_{s} z_{s}$, where the vector $\left(z_{1}, \ldots, z_{s}\right)$ is bilinearly dependent on the vector $\left(x_{1}, \ldots, x_{s}\right)$.

The square of a sum of $s=2^{r}$ squares over any $R$ is again a sum of $s=2^{r}$ squares, i.e. $\left(x_{1}^{2}+\cdots+x_{s}^{2}\right)^{2}=z_{1}^{2}+\cdots+z_{s}^{2}$, where the vector $\left(z_{1}, \ldots, z_{s}\right)$ is bilinearly dependent on the vector $\left(x_{1}, \ldots, x_{s}\right)$.

$[7,(1.8)]$ can be easily generalized to octonion division algebras over fields:

Proposition 3.11. Let $C=\operatorname{Cay}(k, a, b, c)$ be an octonion algebra over a field $k$ with standard involution - . Let $s(k) \geq 8$. If $-a,-b$ and $-c$ are a sum of at most $8^{-1} s(k)$ squares in $k$ then $S_{h}\left(C,{ }^{-}\right)=8^{-1} s(k)$.

Even more generally, we obtain

Proposition 3.12. Let $R$ be a semilocal ring where 2 is invertible.

(1) Let $C=\operatorname{Cay}(R, a)$ be a quadratic étale algebra over $R$ with standard involution - . Let $s(R) \geq 2$. If $-a$ is a sum of at most $2^{-1} s(R)$ squares in $R$, then $S_{h}\left(C,{ }^{-}\right) \leq 2^{-1} s(R)$.

(2) Let $C=\operatorname{Cay}(R, a, b)$ be a quaternion algebra over $R$ with standard involution - . Let $s(R) \geq 4$. If $-a$ and $-b$ are a sum of at most $4^{-1} s(R)$ squares in $R$, then $S_{h}\left(C,{ }^{-}\right) \leq 4^{-1} s(R)$.

(3) Let $C=\operatorname{Cay}(R, a, b, c)$ be an octonion algebra over $R$ with standard involution - Let $s(R) \geq 8$. If $-a,-b$ and $-c$ are a sum of at most $8^{-1} s(R)$ squares in $R$, then $S_{h}(C,-) \leq 8^{-1} s(R)$.

(4) Let $C=\operatorname{Cay}\left(R, a_{1}, \ldots, a_{r}\right), r \geq 2$ be a generalized Cayley-Dickson algebra over $R$ with scalar involution $*$. Let $s(R) \geq 2^{r}$. If $-a_{1}, \ldots,-a_{r}$ are sums of at most $2^{-r} s(R)$ squares in $R$, then $S_{h}(C,-) \leq 2^{-r} s(R)$.

Proof. The proof is analogous to the one given in [7]. We demonstrate how to do (1) for the convenience of the reader: Since $s(R)=2 m$, we can write $-1=\sum_{i=1}^{m} r_{i}^{2}+\sum_{i=1}^{m} s_{i}^{2}$ with $r_{i}, s_{i} \in R$. Let $-a=\sum_{i=1}^{m} t_{i}^{2}$ with $t_{i} \in R$. Thus, in case $\sum_{i=1}^{m} s_{i}^{2}$ is invertible in $R$ there are $v_{1}, \ldots, v_{m} \in C$ such that 
$\left(\sum_{i=1}^{m} t_{i}^{2}\right)\left(\sum_{i=1}^{m} v_{i}^{2}\right)=\sum_{i=1}^{m} s_{i}^{2}$ by Proposition 3.8, and we get

$$
\sum_{i=1}^{m} r_{i}^{2}+\left(\sum_{i=1}^{m} t_{i}^{2}\right)\left(\sum_{i=1}^{m} v_{i}^{2}\right)=\sum_{i=1}^{m}\left(r_{i}^{2}-a v_{i}^{2}\right)=\sum_{i=1}^{m} n_{C}\left(z_{i}\right)
$$

where $z_{i}=\left(r_{i}, v_{i}\right)$. It follows that $S_{h}\left(C,{ }^{-}\right) \leq m$.

There is another way to generalize the idea of the proof of $[7,(1.8)]$, a similar result is mentioned in $[14,(2.1)]$ for the quaternion case:

Proposition 3.13. Let $k$ be a field of $\operatorname{char}(k) \neq 2$, and $C=\operatorname{Cay}(D, c)$ a composition division algebra, i.e. $c \in k^{\times}$and $D$ a quadratic separable field extension or a quaternion algebra. Let ${ }^{-}$be the standard involution of both $D$ and $C$. Assume that $S_{h}(D,-) \geq 2$ and is finite.

(1) If $-c$ is a sum of at most $2^{-1} S_{h}(D,-)$ hermitian squares in $(D,-)$, then

$$
S_{h}\left(C,{ }^{-}\right)=2^{-1} S_{h}(D,-) \text {. }
$$

(2) If $c$ is not a sum of less than or equal to $S_{h}(D,-)$ hermitian squares in $(D,-)$, then

$$
S_{h}(C,-)=S_{h}(D,-)
$$

Also this result can easily be generalized to Cayley-Dickson algebras.

\section{The Hat-Involution}

The hat-involution ${ }^{-}$for quaternion algebras over fields of characteristic not two, used by Lewis [7], can be generalized to our more general setting as follows:

Theorem 4.1. Let $R$ be an arbitrary ring. Let $C$ be a composition algebra over $R$ of rank greater than 2 which is the Cayley-Dickson doubling of a composition algebra $D$ of half its rank, i.e. $C=\operatorname{Cay}(D, P, N)$ as described in 2.2. Let ${ }^{-}$be the standard involution of $D$. Then

$$
\widehat{(u, w)}:=(\bar{u}, w)
$$

is an involution on $C$, which is not scalar.

The proof is a straightforward computation which uses in particular that the multiplication on $C$ is of the form

$$
(u, w)\left(u^{\prime}, w^{\prime}\right)=\left(u u^{\prime}+h\left(w^{\prime}, w\right), w^{\prime} u+w \overline{u^{\prime}}\right)
$$

for $u, u^{\prime} \in D$ and $w, w^{\prime} \in P$, and where $h: P \times P \rightarrow D$ is the ${ }^{-}$-hermitian form induced by the norm $N: P \rightarrow R$ (written as unique bilinear map $P \times P \rightarrow D$ which satisfies the identity $(w u)(w v)=N(w) \bar{v} u$ in $[9,(2.5)])$. We call this involution the hat-involution. Note that by the definition of the hat-involution,

$$
S_{h}(C, \uparrow) \leq S_{h}(D,-)
$$

A straightforward computation shows that $\operatorname{Sym}(C, \widehat{ })$ is a projective $R$-module of (constant) rank 3 and $\operatorname{Skew}(C, \widehat{)})$ a projective $R$-module of (constant) rank 
1 if $C$ is a quaternion algebra. If $C$ is an octonion algebra, then $\operatorname{Sym}\left(C,{ }^{\wedge}\right)$ is projective of (constant) rank 5 , and $\operatorname{Skew}(C, \widehat{ })$ of (constant) rank 3.

Theorem 4.2 ([11]). Let $R$ be a ring with $2 \in R^{\times}$, and $C$ a composition algebra of rank $r$ over $R$. There is a one-one correspondence between non-standard involutions of the first kind on $C$ and composition subalgebras of rank $r / 2$. More precisely:

(1) Let $\tau$ be an involution on $C$ of the first kind which is not the standard involution - . Then

$$
C \cong \operatorname{Cay}(B, P, N)
$$

with $B=\{x \in C \mid \tau(x)=\bar{x}\}$ a composition subalgebra of $C$, and with $P=\{x \in C \mid \tau(x)=-\bar{x}\}$.

(2) If $B$ is a composition subalgebra of $C$ of rank $r / 2$, then $B$ determines a nontrivial automorphism $f \in \operatorname{Aut}(C)$ satisfying $f^{2}=\mathrm{id}$ and thus an involution $\tau=f \circ^{-}$of the first kind.

This implies that there exist composition algebras whose only involution of the first kind is the standard one. This is because not every quaternion or octonion algebra over a ring contains a composition subalgebra of half its rank. For examples, the reader is referred to [5], where octonion algebras over polynomial rings are constructed, which indeed only have a composition subalgebra of rank 1. See also [10].

Proposition 4.3 ([11]). Let $R$ be a ring with $2 \in R^{\times}$, and let $C$ be a composition algebra over $R$ with non-standard involution $\tau$ of the first kind. Then $C \cong \operatorname{Cay}(B, P, N)$ and $\tau((u, v))=(\bar{u}, v)$ is the hat-involution on $\operatorname{Cay}(B, P, N)$.

As an immediate consequence it follows that in order to compute the hermitian level of a composition algebra $C$ with arbitrary non-standard involution, it suffices to find the values of $S_{h}(C, \widehat{\imath})$.

Lemma 4.4. Let $R$ be a ring with $2 \in R^{\times}$, and $C$ a split composition algebra over $R$, equipped with the hat-involution. If $C=\operatorname{Cay}(D, P, N)$ with $D$ split, or if $C=\operatorname{Cay}(D, \mu)$ with $\mu \in R^{\times 2}$, then $S_{h}(C, \widehat{ })=1$.

Proof. If $C=\operatorname{Cay}(D, P, N)$ with $D$ a split composition algebra over $R$, then

$$
S_{h}(C, \widehat{ }) \leq S_{h}(D,-)=1
$$

If $C=\operatorname{Cay}(D, \mu)$ for some $\mu \in R^{\times 2}$ with $D$ not necessarily split, then there exists an element $v \in C$ such that $\bar{v} v=-1$, and such that $\widehat{(0, v)}(0, v)=(\bar{v} v, 0)=$ $(-1,0) \in D \oplus D=C$.

The statement of $[7,(2.2)]$ also holds if $C$ is an octonion algebra: 
Lemma 4.5. Let $k$ be a field of $\operatorname{char}(k) \neq 2$. If $C=\operatorname{Cay}(k, a, b, c)$ is a composition division algebra over $k$, and $\mathrm{Cay}(k, a, b,-c)$ is not a division algebra, then

$$
S_{h}(C, \widehat{)})=1
$$

Proof. Put $D=(a, b)_{k}$. Then $D$ is a division algebra, and Cay $(k, a, b,-c)$ is no division algebra if and only if there is an element $z \in D^{\times}$such that $-c=\bar{z} z$. View $z$ as an element in $C$, and let $1, i, j, k, e$ be a standard basis of $C$, then $\widehat{z} z=$ $\bar{z} z=-c$. We compute $\widehat{\left(z e^{-1}\right)}\left(z e^{-1}\right)=c^{-2}(e \bar{z})(z e)=c^{-2}(z e)(z e)=c^{-2} c \bar{z} z=-1$ using the well-known identities $e \bar{z}=z e$ and $(u e)(v e)=c \bar{v} u$ for $u, v \in D$.

In particular, for any field where there is only one octonion division algebra $C$, it follows that $S_{h}(C, \widehat{\wedge})=1$ (cf. $[7,(2.3)]$ ).

Example. Let $C=\operatorname{Cay}(\mathbb{R},-1,-1,-1)$ be the real octonions. Then $S_{h}(C, \widehat{ })=1$.

Proposition 4.6. Let $C=\operatorname{Cay}(D, P, N)$ be a composition algebra over a ring $R$ where 2 is an invertible element. Define $\widetilde{C}=\operatorname{Cay}(D, P,-N)$.

(1) $S_{h}(\widetilde{C},-) \leq S_{h}(C, \widehat{\imath})$.

(2) If $m=S_{h}(\widetilde{C},-)$ and if there are elements $x_{i}=\left(u_{i}, w_{i}\right) \in \widetilde{C}$ such that both $-1=\sum_{i=1}^{m} \bar{x}_{i} x_{i}$ and $0=\sum_{i=1}^{m} w_{i} \bar{u}_{i}$ for $x_{i}=\left(u_{i}, w_{i}\right) \in \widetilde{\widetilde{C}}$, then $S_{h}(C, \widehat{ }) \leq S_{h}(\widetilde{C},-)$ as well, and in particular, $S_{h}(C, \widehat{ })=S_{h}(\widetilde{C},-)$.

Proof. For an element $x \in C=\operatorname{Cay}(D, P, N)$ with $x=(u, w)$ we compute

$$
\widehat{x} x=(\bar{u}, w)(u, w)=(\bar{u} u+h(w, w), w \bar{u}+w \bar{u})=\left(n_{D}(u)+N(w), 2 w \bar{u}\right) .
$$

Thus we obtain for $x_{i}=\left(u_{i}, w_{i}\right) \in C$ that

$$
\left.-1=\sum_{i=1}^{m} \widehat{x_{i}} x_{i}=\sum_{i=1}^{m} \widehat{\left(u_{i}, w_{i}\right.}\right)\left(u_{i}, w_{i}\right)=\sum_{i=1}^{m}\left(n_{D}\left(u_{i}\right)+N\left(w_{i}\right), 2 w_{i} \bar{u}_{i}\right)
$$

which is equivalent to

$$
-1=\sum_{i=1}^{m}\left(n_{D}\left(u_{i}\right)+N\left(w_{i}\right)\right) \quad \text { and } \quad 0=\sum_{i=1}^{m} w_{i} \bar{u}_{i} .
$$

The first equality, however, is equivalent to

$$
-1=\sum_{i=1}^{m} n_{\widetilde{C}}\left(\left(u_{i}, w_{i}\right)\right)=\sum_{i=1}^{m} \overline{\left(u_{i}, w_{i}\right)}\left(u_{i}, w_{i}\right)
$$

where ${ }^{-}$is the standard involution on $\widetilde{C}$.

This generalizes an observation by Serhir [14, Sec. 4.2].

Proposition $4.7([7,(2.4)])$. For any power of two, $n=2^{\ell}$, there exists an octonion division algebra $C$ over a field such that $S_{h}(C, \widehat{)})=2^{\ell-1}$. 
Proof. The proof is analogous to the proof of $[7,(2.4)]$. Take

$$
k=\mathbb{R}\left(x_{1}, \ldots, x_{n}\right)\left(\sqrt{-\sum_{i=1}^{n} x_{i}^{2}}\right)
$$

with $n=2^{\ell}$. Then $s(k)=n$. Consider the rational function field $K=k\left(t_{1}, t_{2}\right)$ in two variables over $k$ and the octonion division algebra $C=\operatorname{Cay}\left(K,-1,-t_{1},-t_{2}\right)$. Obviously, $m:=S_{h}(C, \widehat{ }) \leq S_{h}\left(k(\sqrt{-1}),{ }^{-}\right)=2^{\ell-1}$. A straightforward computation shows that $0=\sum_{i=1}^{m+1} \widehat{x_{i}} x_{i}$ implies

$$
0=\sum_{i=1}^{m+1} p_{i}^{2}+q_{i}^{2}+t_{1}\left(r_{i}^{2}+s_{i}^{2}\right)+t_{2}\left(\alpha_{i}^{2}+\beta_{i}^{2}+t_{1}\left(\gamma_{i}^{2}+\delta_{i}^{2}\right)\right)
$$

for $x_{i}=p_{i}+q_{i} i+r_{i} j+s_{i} k+\alpha_{i} e+\beta_{i} i e+\gamma_{i} j e+\delta_{i} k e$ with $p_{i}, q_{i}, r_{i}, s_{i}, \alpha_{i}, \beta_{i}, \gamma_{i}, \delta_{i} \in K$. We may assume without loss of generality that these are all elements in $k\left[t_{1}, t_{2}\right]$. Put $t_{1}=t_{2}=0$, then we obtain a sum of $(2 m+2)$ squares in $k$. Thus $s(k) \leq 2 m+1$ so that $2^{\ell} \leq 2 m+1$, i.e. $m \geq 2^{\ell-1}-1 / 2$. Since $m$ is an integer we must have $m=2^{\ell-1}$.

It is an interesting question which values indeed are possible for the hermitian level $S_{h}(C, \widehat{ })$ of an octonion algebra $C$. For a quaternion algebra $D$ over a field of characteristic not 2 , it is known that $S_{h}(D, \widehat{)})$ is always a power of two, provided that it is finite [14, Corollaire 3.2].

Lemma 4.8. Let $k$ be a field of characteristic not two such that $s(k)=2^{t} \geq 8$. Let $C=\operatorname{Cay}(k, a, b, c)$ be an octonion division algebra.

(1) If $-a,-b$ and $c$ are each a sum of at most $8^{-1} s(k)$ squares in $k$, then

$$
2^{t-3} \leq S_{h}(C, \widehat{ }) \leq 2^{t-2} \text {. }
$$

(2) If $-c$ is not a sum of less than or equal to $S_{h}(D,-)$ hermitian squares in $(D,-)$, and if $\widetilde{C}=\operatorname{Cay}(D,-c)$ is a division algebra then $S_{h}(C, \widehat{ })$ is a power of 2 .

Proof. (1) For $\widetilde{C}=\operatorname{Cay}(k, a, b,-c)$ we have $S_{h}(\widetilde{C},-)=2^{t-3}=2^{-1} S_{h}(D,-)$ with $D=(a, b)_{k}$. Therefore the assertion follows from the fact that $S_{h}(\widetilde{C},-) \leq$ $S_{h}(C, \widehat{ }) \leq S_{h}(D,-)$. $\tilde{C}$.

(2) In this case, $S_{h}(\widetilde{C},-)=S_{h}(D,-)$ by Proposition 3.13 applied to the algebra

Remark 4.9. Let $C=\operatorname{Cay}(k, a, b, c)$ be an octonion division algebra over a field. For $x_{i}=p_{i}+q_{i} i+r_{i} j+s_{i} k+\alpha_{i} e+\beta_{i} i e+\gamma_{i} j e+\delta_{i} k e$ with $p_{i}, q_{i}, r_{i}, s_{i}, \alpha_{i}, \beta_{i}, \gamma_{i}, \delta_{i} \in k$ we calculate that $0=\sum_{i=1}^{m} \widehat{x_{i}} x_{i}$ is equivalent to

$$
0=\sum_{i=1}^{m}\left(p_{i}^{2}-a q_{i}^{2}-b r_{i}^{2}+a b s_{i}^{2}\right)-c\left(\alpha_{i}^{2}-a \beta_{i}^{2}-b \gamma_{i}^{2}+a b \delta_{i}^{2}\right)
$$


and

$$
0=\sum_{i=1}^{m}\left(\alpha_{i}+\beta_{i} i+\gamma_{i} j+\delta_{i} k\right)\left(p_{i}-q_{i} i-r_{i} j-s_{i} k\right) .
$$

Now (2) is equivalent to the equations

$$
\begin{aligned}
& 0=\sum_{i=1}^{m}\left(\alpha_{i} p_{i}-\beta_{i} q_{i} \alpha-\gamma_{i} r_{i} b+a b \delta_{i} s_{i}\right) \\
& 0=\sum_{i=1}^{m}\left(-\alpha_{i} q_{i}+\beta_{i} p_{i}+\gamma_{i} s_{i} b-\delta_{i} r_{i} b\right) \\
& 0=\sum_{i=1}^{m}\left(\alpha_{i} r_{i}-\beta_{i} s_{i} a+\gamma_{i} p_{i}+\delta_{i} q_{i} a\right) \\
& 0=\sum_{i=1}^{m}\left(-\alpha_{i} s_{i}-\beta_{i} r_{i}+\gamma_{i} q_{i}+\delta_{i} p_{i}\right) .
\end{aligned}
$$

Thus 0 is a nontrivial sum of $m$ hermitian squares in $(C, \widehat{ })$ if and only if in $k$ there exists a nontrivial common zero of 5 quadratic forms in $8 m$ variables

$$
\begin{gathered}
p_{1}, \ldots, p_{m}, q_{1}, \ldots, q_{m}, s_{1}, \ldots, s_{m}, r_{1}, \ldots, r_{m}, \\
\alpha_{1}, \ldots, \alpha_{m}, \beta_{1}, \ldots, \beta_{m}, \gamma_{1}, \ldots, \gamma_{m}, \delta_{1}, \ldots, \delta_{m} .
\end{gathered}
$$

This indicates that it may be quite difficult to determine which values can occur as $S_{h}\left(C,{ }^{\wedge}\right)$.

$[7,4.1,4.2]$ generalize to octonion algebras as follows:

Lemma 4.10. Let $k$ be a formally real field and $C=\operatorname{Cay}(k, a, b, c)$ an octonion algebra over $k$.

(1) Let $S_{h}(C,-)<\infty$.

(a) If $-a$ and $-b$ are sums of squares in $k$ then so is $c$.

(b) If $-c$ and $-a($ or $-b)$ are sums of squares in $k$ then so is $b$ (or $a)$.

(2) Let $S_{h}(C, \widehat{\imath})<\infty$.

(a) If $-a$ and $-b$ are sums of squares in $k$ then so is $-c$.

(b) If $c$ and $a$ (or b) are sums of squares in $k$ then so is $-b$ (or $-a)$.

Proof. (1) The proof is analogous to the one of [7, 4.1].

(2) If $S_{h}(C, \widehat{\imath})<\infty$ then $S(\widetilde{C},-)<\infty$ for $\widetilde{C}:=\operatorname{Cay}(k, a, b,-c)$. Hence (1) implies the assertion.

Lemma 4.11 ((cf. $[7,4.3]))$. Let $k$ be a formally real field, and $C=\operatorname{Cay}(k, a, b, c)$ an octonion algebra over $k$. If both $-a$ and $-b$ are sums of squares in $k$ then either $S_{h}\left(C,{ }^{-}\right)$or $S_{h}(C, \uparrow)$ is infinite.

The proof is again analogous to the one given in [7]. 
Conjecture. Let $k$ be a field of characteristic not two and let $C$ be any octonion division algebra over $k$. If $S_{h}(C, \widehat{)})$ is finite, it must be a power of two.

\section{Involutions of the Second Kind}

We now briefly turn to involutions of the second kind on composition algebras. Involutions of the second kind on generalized Cayley-Dickson algebras and composition algebras behave special in the following way. (For quaternion algebras, this result is due to Albert, cf. [6, I.2.(2.22)].)

Theorem 5.1 ([11]). Suppose 2 is invertible in $R$. Let $S$ be a quadratic étale faithfully flat $R$-algebra with standard involution $s_{0}$. Let $C$ be a composition algebra over $S$ of rank $\geq 4$, and $A=\operatorname{Cay}\left(C, \mu_{1}, \ldots, \mu_{m}\right)$ a generalized CayleyDickson algebra of $\operatorname{rank} A=2^{m} \operatorname{rank} C \geq 16$ (i.e. A is a noncommutative Jordan algebra). Let $\tau$ be any involution on $A$ of the second kind.

(1) There exists a unique flexible quadratic $R$-subalgebra $A_{0}$ of $A$ with a unique scalar involution $\sigma_{0}$ such that

$$
A=A_{0} \otimes_{R} S \quad \text { and } \quad \tau=\sigma_{0} \otimes s_{0} .
$$

The algebra $A_{0}$ is uniquely determined by these conditions.

(2) There exists a unique composition $R$-subalgebra $C_{0}$ of $C$ such that

$$
A=C_{0} \otimes_{R} S \quad \text { and } \quad \tau=-\otimes s_{0},
$$

where ${ }^{-}$denotes the standard involution on $C_{0}$. The algebra $C_{0}$ is uniquely determined by these conditions.

We restrict ourselves to the study of these involutions on composition algebras, since it is immediately obvious how to obtain an analogous statement also for generalized Cayley-Dickson algebras. In the above situation, let $\tau$ be an involution of the second kind on a composition algebra $C$ over a quadratic étale $R$-algebra $S$. We know that there is a unique composition subalgebra $C_{0}$ over $R$ such that $C \cong C_{0} \otimes_{R} S$ and $\tau=-\otimes s_{0}$. Obviously,

$$
S_{h}(C, \tau) \leq \min \left\{S_{h}\left(C_{0},-\right), S_{h}\left(S, s_{0}\right)\right\}
$$

Moreover, if the quadratic étale algebras $S=\operatorname{Cay}(R, c)$ is a classical CayleyDickson doubling of $R$, write $x=(z, w) \in C=C_{0} \oplus C_{0}$ (as an $S$-module) with $z, w \in C_{0}$. We compute $\tau(x)=(\bar{z},-\bar{w})$ and

$$
\tau(x) x=\left(n_{C_{0}}(z)-c n_{C_{0}}(w),-(\bar{z} w+\bar{w} z)\right)=\left(n_{C_{0}}(z)-c n_{C_{0}}(w),-n_{C_{0}}(z, w)\right) .
$$


Thus

$$
\begin{aligned}
-1 & =\sum_{i=1}^{m} \tau\left(x_{i}\right) x_{i} \\
& =\left(\sum_{i=1}^{m}\left(n_{C_{0}}\left(z_{i}\right)-c n_{C_{0}}\left(w_{i}\right)\right),-\sum_{i=1}^{m} n_{C_{0}}\left(z_{i}, w_{i}\right)\right) \\
& =\left(\sum_{i=1}^{m} n_{A}\left(\left(z_{i}, w_{i}\right)\right),-\sum_{i=1}^{m} n_{C_{0}}\left(z_{i}, w_{i}\right)\right)
\end{aligned}
$$

with $A:=\operatorname{Cay}\left(C_{0}, c\right)$ the generalized Cayley-Dickson algebra over $R$, and $\left(z_{i}, w_{i}\right) \in$ A.

Lemma 5.2. In the above situation,

$$
S_{h}(A, *) \leq S_{h}(C, \tau) \leq S_{h}\left(C_{0},-\right) .
$$

If $S / R=k / k_{0}$ is a separable quadratic field extension, then

$$
d_{1} \leq 2 S_{h}(C, \tau) \leq d_{2}
$$

where $d_{1}$ is the order of the quadratic form $n_{A}$ in $W(k)$, and $d_{2}$ the order of the quadratic form $n_{C_{0}}$ in $W(k)$.

In particular, if $c$ is not a sum of less than or equal to $S_{h}\left(C_{0},-\right)$ hermitian squares in $\left(C_{0},-\right)$ then $S_{h}(C, \tau)$ is a power of two.

Proof. We know that $S_{h}\left(C_{0},{ }^{-}\right)=S_{h}(A, *)$ by Proposition 3.13 (or, more precisely, its generalization for Cayley-Dickson algebras). Thus the assertion follows from $S_{h}(A, *) \leq S_{h}(C, \tau) \leq S_{h}\left(C_{0},-\right)$ (Proposition 3.5).

\section{ACKNOWLEDGEMENTS}

The second author thanks the first author and the Fakultät für Mathematik, Universität Regensburg, for hospitality while this research was undertaken. Part of this research is funded by the TMR research network (ERB FMRX CT-970107) on " $K$-theory and algebraic groups".

\section{REFERENCES}

[1] Baeza, R: Quadratic Forms over Semilocal Rings, Lecture Notes in Mathematics, vol. 655, Springer-Verlag, Berlin-Heidelberg-New York, 1978

[2] Dai, Z.D., Lam, T.Y., Milgram, R.J.: Application of topology on problems of sums of squares, Enseign. Math. (2) 27, 277-283 (1981)

[3] Jacobson, N.: Composition algebras and their automorphisms, Rend. Circ. Mat. Palermo (2) 7, 55-80 (1958)

[4] Knebusch, M: Runde Formen über semilokalen Ringen, Math. Ann. 193, 21-34 (1971)

[5] Knus, M.-A., Parimala, R., Sridharan, R.: On compositions and triality, J. Reine Angew. Math. 457, 45-70 (1994)

[6] Knus, M.-A., Merkurjev, A., Rost, M., Tignol, J.-P.: The Book of Involutions, AMS Coll. Publications, Vol. 44 (1998) 
[7] Lewis, D.W.: Sums of hermitian Squares, J. Algebra 115, 466-480 (1998)

[8] McCrimmon, K.: Nonassociative algebras with scalar involution, Pacific J. Math. 116 (1), 85-109 (1985)

[9] Petersson, H.: Composition algebras over algebraic curves of genus 0, Trans. Amer. Math. Soc. 337, 473-493 (1993)

[10] Pumplün, S.: Quaternion algebras over elliptic curves, Comm. Algebra, 26 (12), 43574373 (1998)

[11] Pumplün, S.: Involutions on composition algebras, preprint.

[12] Schafer, R.D.: An Introduction to Nonassociative Algebras, Corrected reprint of the 1966 original, Dover, New York, 1995

[13] Scheiderer, C.: On sums of squares in local rings, J. Reine Angew. Math. 540, 205-227 (2001)

[14] Serhir, A.: Niveau hermitien de certaines algèbres de quaternions, Comm. Algebra 25 (8), 2531-2538 (1997)

S. Pumplün, FAkultät Für Mathematik, Universität Regensburg, UniversitätsStr. 31, D-93040 Regensburg, Germany.

E-mail address: susanne.pumpluen@mathematik.uni-regensburg.de

T. Unger, Department of Mathematics, University College Dublin, Belfield, Dublin 4, Ireland.

E-mail address: thomas.unger@ucd.ie 